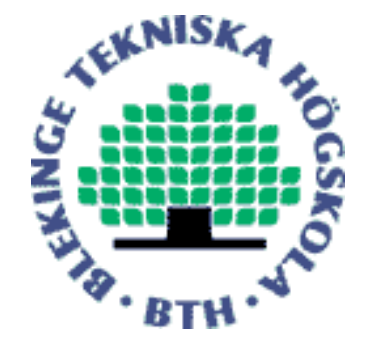

Copyright (C) 2010 IEEE.

Citation for the published paper:

A Multi-Reality Approach to CRN Data Representation

Alexandru Popescu, David Erman, Markus Fiedler, Demetres Kouvatsos

1st International Workshop on System and Theory of Cognitive Radio Networks

2010 Hangzhou, China

This material is posted here with permission of the IEEE. Such permission of the IEEE does not in any way imply IEEE endorsement of any of BTH's products or services Internal or personal use of this material is permitted. However, permission to reprint/republish this material for advertising or promotional purposes or for creating new collective works for resale or redistribution must be obtained from the IEEE by sending a blank email message to pubs-permissions@iee.org.

By choosing to view this document, you agree to all provisions of the copyright laws protecting it. 


\title{
A Multi-Reality Approach to CRN Data Representation
}

\author{
Alexandru Popescu ${ }^{\dagger \ddagger}$, David Erman ${ }^{\dagger}$, Markus Fiedler ${ }^{\dagger}$ and Demetres Kouvatsos ${ }^{\ddagger}$ \\ ${ }^{\dagger}$ Dept. of Telecommunication Systems \\ School of Engineering \\ Blekinge Institute of Technology \\ 37179 Karlskrona, Sweden \\ $\ddagger$ Dept. of Computing \\ School of Informatics \\ University of Bradford \\ Bradford, West Yorkshire BD7 1DP, United Kingdom
}

\begin{abstract}
-
Cognitive Radio Networks (CRNs) are emerging as a viable solution for exploiting the available spectrum and solving overcrowding in the operating bands. Several dimensions in the process of identification, management and routing need to be handled to overcome the challenges associated with the management of CRNs. A very important topic in achieving an effective and flexible management solution is related to the data representation of the different CRN dimensions. Scalability, high tolerance to churn and low latencies in accessing the stored data are fundamental characteristics in order to keep up with the dynamic nature of a mobile environment. Accordingly, to achieve a robust data representation that can handle all these requirements in an efficient way we focus our attention on peer-to-peer (P2P) systems. These systems are well known for providing an efficient platform to develop scalable and robust applications. Hence, in this paper we investigate using geometric multi-dimensional data representation and addressing system based on basic CAN functionality. Results obtained in our experiments are reported, providing useful information to improve the resilience to churn of our system.
\end{abstract}

\section{INTRODUCTION}

Over the last years we have seen a tremendous increase in the amount of wireless services and devices. The mobile phones of today are for instance little more than miniaturized computers incorporating much the same versatility and functionality. Obviously, the way in which these devices are used has also been fundamentally changed, people today are simply used to always being connected and instantly have access to information.

However, this development is now revealing the fundamental limitations and inflexibility of current designs for wireless communication technologies. Densely populated areas already suffer from an overcrowding in the operating bands. Furthermore, given that the electromagnetic spectrum is a limited resource this problem is going to intensify during the next few years due to the explosion in the amount of mobile devices.

To amend some of these issues CRNs are emerging as a viable solution [1]. By configuring the transmission parameters of a Cognitive Radio Device (CRD), adaptations can be made to the surrounding environment and exploiting every part of available spectrum [2].
Furthermore, having devices able to adapt and to learn from experience opens up for new opportunities. Innovative solutions can be developed to facilitate the use of mobile devices, which can adapt on-the-fly and use whatever resources are available at the moment.

Today, mobile operators are forced to utilize very small portions of the available spectrum, limiting so the number of users that can be serviced simultaneously by a single Base Station (BS). This constraint increases the probability for blockage in densely populated cells. Up to now a solution to increase the network capacity in highly populated areas has been to introduce additional picocells [3], [4]. Yet another solution has revealed itself with the emerging of Cognitive Radio.

By implementing a particular Cognitive Radio architecture, as suggested in [2], at every base station and mobile device, operators are presented with an opportunity to increase the resource utilization and reduce so the blockage probability. An important part of the suggested architecture consists of a highly modified and adapted Multi-Dimensional ContentAddressable Network (MD-CAN) [2], which is used for:

- Describing the CRN topology (including free holes represented as CAN zones).

- Data representation of operational parameters per CRD.

- End-to-end (e2e) path computation for communicating CRDs.

The dimensions we use are space, frequency, power, code and cost. Additional dimensions can of course be used like, e.g., angle (referring to the beam direction) and elevation (referring to the height above ground level).

Consequently, it is very important to have good simulation tools to test and analyze realistic scenarios. The paper focuses therefore on the implementation of an adapted multi-reality CAN simulator for use in our suggested CRN architecture. Having network members consisting of mobile nodes, a particular focus is given to the end-to-end (e2e) path computation, availability and routing success in case of massive amounts of churn. Preliminary results obtained in our experiments are reported as well as future work and developments.

The rest of the paper is organized as follows. In section II we provide a short overview and introduction to the MD-CAN 
data representation. In section III we discuss the implementation of our multi-reality data-representation for the CRN space dimension. The simulation results are described in Sections IV and finally, section $\mathrm{V}$ concludes the paper.

\section{SN DATA REPRESENTATION}

The suggested architecture reported in [2] is used to control the fundamental operations in the management of a CRN. To achieve this, a centralized entity called Support Node (SN) was introduced to provide support for the cognitive radio communication of associated CRDs. The $\mathrm{SN}$ also presents the mean to prioritize among unlicensed users competing for free spaces, allowing for scheduling of resource distribution among arriving unlicensed users.

In short, many vital operations for the functionality of the suggested CRN architecture are provided by the SN. As such, to cope with these requirements, the SN needs an optimal way to structure all received information in order to update and access it quickly. To achieve a robust data representation that can handle massive amounts of information in an efficient way, we turn our attention towards peer-to-peer (P2P) systems. These systems are well-known for providing an efficient platform to develop scalable and robust applications.

In this paper, we are particularly interested in the ContentAddressable Network system and, specifically, the multidimensional version of it. In regular CAN implementation, member nodes have $\Theta(2 d)$ neighbors and the average path lengths are of $\Theta(d / 4)\left(n^{1 / d}\right)$ hops (where $d$ is the number of dimensions and $n$ is the number of nodes) [5]. The key space is an $n$-dimensional Cartesian coordinate space that wraps around the edges of each dimension, thus creating a $n$ dimensional torus geometry. Nodes in the CAN are responsible for their own zone (a portion of the key space) and are identified by a point $P$ in the key space. Additionally, every node has information about adjacent zones and their responsible nodes that also provides the means to route messages amongst the CAN member nodes.

By assuming a regular MD-CAN where every node keeps track of its own neighbors, we have a flexible solution to adapt to our needs. Hence, for data representation and addressing purposes in a multihop CRN we use a modified MD-CAN architecture. The addressing data of the CRN is stored at the SN and represented through an MD-CAN. Different CAN dimensions can be used to represent different CR-dimensions like, e.g., space, frequency, power, code. A hole in a CRdimension at a specific time can be represented as a vacant zone in a particular CAN dimension. This adapted functionality will no longer conform to regular MD-CAN operations. It can instead be viewed as a geometric multi-dimensional data representation and addressing system based on basic CAN functionality.

Stored operational parameters for a node in different CR-dimensions are identified with the Cartesian coordinates $n(t)=(x(t), y(t), f(t), p(t))$, where $x(t)$ and $y(t)$ denote the space dimension (current CRN topology), $p(t)$ the power dimension and $f(t)$ the frequency dimension (partitioned into channels, $c \leftarrow f(t))$. All parameters are functions of time, meaning that $n(t)$ can, at any given time, characterize a CRD occupying the operational zone at coordinates $x, y$ in the space dimension, channel $c$ in the frequency dimension and using the transmission output power $p$ in the power dimension [2]. Each dimension has its own defining characteristics that can be changed independently of each other for optimization purposes from the perspective of every CR user. Simply put, a user might need to adapt in one or more CR-dimensions to keep the location in the space dimension. A CRD wanting to communicate with a particular destination according to specific QoS constraints always needs to send a path computation request to the local SN. By having a complete overview of the available resources and network topology, the $\mathrm{SN}$ can then compute an e2e communication path, which guarantees the specific QoS constraints of the CRD.

Finding an appropriate path between a specific source and destination in a CRN is very challenging [6]. Routing is done while having several CR-dimensions that need to be simultaneously managed by considering the variations in the available frequency bands and topology. Naturally, due to churn and node mobility, destinations might become unreachable leading so to invalid paths and routing breakdown. In other words, we need to devise a way of improving the success rates of the path computations even in the presence of massive amounts of churn. One way to achieve this is to use a multireality approach for storing the space dimension information, meaning the CRN topology. Multi-realities are generally used as a way to reduce path lengths, though another important benefit of this approach is the improved data availability.

Several independent coordinate spaces are maintained with each node in the system for every reality, meaning the content of the hash table is replicated in each reality. Hence the routing fault tolerance is improved. Structured P2P systems like CAN have their architecture based on using a distributed hash table (DHT) layer. The node identification is mapped in this layer (or key space) and information about data objects is placed at peers identified by the key of the specific data object [5].

If a destination becomes unavailable in one reality it might still be reachable in another reality where a different path can be computed. Furthermore, having member nodes handling a zone (with a different set of coordinates) per reality means that forwarding a message becomes a matter of choosing the reality with the shortest distance to the destination. In regular multireality CAN implementation every node is assigned an arbitrarily selected additional zone in each coordinate space, which is called a reality. However, adapting this approach to mobile nodes infers the need to account for geographical distances between the assigned zones (per node) in every particular reality. Hence, a particular node can only be assigned zones and neighbors that are reachable from its current geographical location and within its available transmit power output. For this reason the CAN realities in our approach do not wrap around the edges of every dimension to create a torus geometry.

A node in one corner of the CRN space dimension (represented as one dimension in the n-dimensional Cartesian coor- 
dinate space), might not be able to handle a zone in another corner of the coordinate space due to practical limitations. The intermediate physical distance between the two might be too far or the transmit power output might simply not be enough. Because of this, an approach is chosen where the Cartesian space (for the space dimension) is split into four equally sized parts.

\section{IMPLEMENTING A MULTI-REALITY DATA} REPRESENTATION FOR THE CRN SPACE DIMENSION

A typical scenario for an e2e path computation begins when a CRD wishes to communicate with another CRD in the local CRN. The corresponding SN is first contacted for compiling a valid path between the communicating CRD and the destination. A lookup in the SNs member table identifies the locations of both the source and the destination. Once identified, the SN compiles a path between the two. It starts by checking the available space dimension realities for a valid path.

For now, the routing path is computed by checking all available space dimension realities and selecting the one with the shortest distance between source and destination. The computed path is used by the communicating CRD to accordingly route its messages to the destination. However, since every member node has parameters for every CRN dimension associated with it, we plan as future work on expanding the functionality of our multi-reality approach to consider many other factors from all CRN dimensions. This means that the capability of computing paths is increased according to user preferences and environmental constraints like maximum power output, cost and throughput.

Naturally, working with a mobile environment entails the need to consider massive amounts of churn and the impact it has on our suggested management approach. A general definition of churn is a process that characterizes the node arrival and departure in an overlay network. Two types of departures are possible, either graceful leave or due to node failure. This is an important phenomenon that usually has serious impacts on the performance of a network, e.g., routing failures, data inconsistency and increased delay and jitter [7], [8].

Furthermore, this also means that networks must be able to tolerate failures as they occur and be able to recover from their effects. Given a worst case scenario of churn presence, the goal is to implement and evaluate the performance of several important algorithms to increase the robustness and resilience of our data representation used for the CRN management. We aim at devising a way of improving the success rates of the end-to-end (e2e) path computations in our suggested CRN management approach, even though massive amounts of churn is present.

In this paper we assume churn to be a global process where nodes join and leave the network [7], [9]. The process of selecting the churned nodes is selected to be Random uniform among all active nodes. Furthermore, the elapsed time from when a specific node first joins until its final leave is considered to be a node lifetime. The global join process is usually modeled by a Poisson process, whereas the leave process is modeled in different ways depending upon the type of leave.

In general, after completion of the off time, the churned node rejoins the network. However, in our case if a node has entered an off state it must rejoin the network as a new arrival in order to avoid locking down valuable resources. Hence, once a node has entered the off state its resources are released and distributed amongst unlicensed users waiting to receive service and be scheduled by the SN. However, given that in this paper we only consider the worst case scenarios, when a node enters the off state it not only entails that the end of its lifetime has been reached but also that no new arrivals are available to take its place. Naturally, gaps and inconsistencies are then created in the network leading to routing failures. The goal is to implement and evaluate a multi-reality CAN construction algorithm for the CRN space dimension through simulation. By increasing the number of realities and monitoring the fault tolerance for different network sizes we can conclude the optimal number of realities to use depending on the network size.

Having our solution based on an adapted CAN approach means that every CRD member in the CRN space dimension is identified by a point $P$ and it is responsible for its own zone. Member nodes only have information about adjacent neighbors and messages are routed in much the same way as in a regular CAN implementation. The shortest path from source to destination is selected and messages are forwarded in a greedy manner [10]. Naturally, the shortest path is in this case compiled and optimized with regard to user preferences and environmental constraints [11]-[13]. Furthermore, the e2e routing can be done in a one-dimensional space by mapping the associated QoS metrics from the multi-dimensional space present in a CRN [14]-[16].

Our implementation creates a 2-dimensional $[0,1] \times[0,1]$ coordinate space for the CRN space dimension. Due to physical constraints of the member CRDs the dimensions are not wrapped around the edges to create a torus geometry. Instead, the space dimension spans over a certain geographical area. The coordinate space is populated according to a scheduler to fairly partition the spectrum holes between the secondary users. In other words, bootstrapping is handled by the actual $\mathrm{SN}$, which handles requests from unlicensed users wishing to join the CRN [2], [17].

As previously mentioned, the space dimension is split into four equally sized pieces. Each quarter is maintained throughout every stored reality and the maximum spanning radius of every quarter is selected according to predetermined constraints. All CRDs in each quarter of the Cartesian space are assumed to be interchangeable, meaning be able to handle whatever zone they are assigned in that particular area of the coordinate space, as shown in figure 1.

When the space dimension has been populated, a fully operational CRN has been achieved. The network is managed and kept updated with occurring changes through a number of 


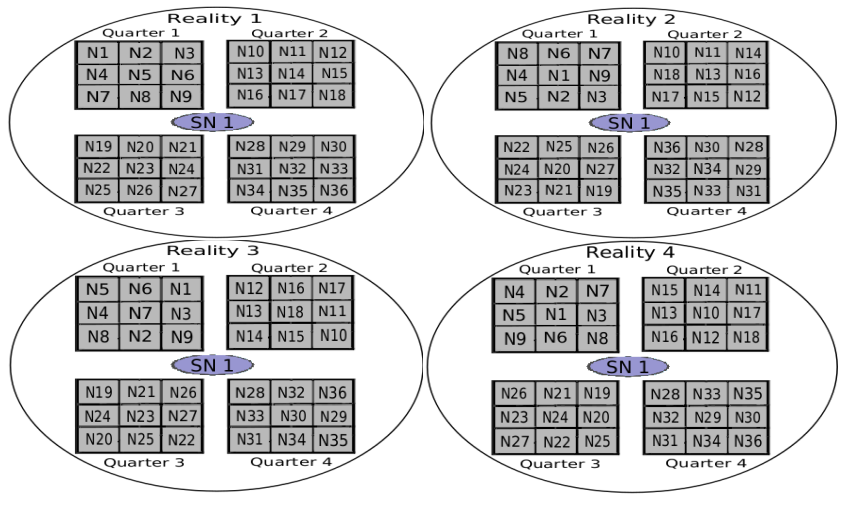

Fig. 1. Reality quarters.

control algorithms as discussed in [2].

\section{SiMUlation EXPERIMENTS}

In our current implementation we focused on simulating the success rates of communications occurring after the CRN has been populated. We begin by simulating a single reality with a predetermined number of nodes $n$. Once the coordinate space is populated, 10 nodes are selected to communicate with 10 other member nodes in the network. Although, before communications are allowed to start a churn percentage $p$ is introduced. Random nodes in the network (up to the particular churn percentage) are hence rendered inert leaving the network with obvious inconsistencies.

Two experiments have been made, one with a single simulated reality and the other with multiple realities simulated. The experiments are run 100 times each while storing the number of communication failures per run. Each experiment is repeated with increasing number of nodes undergoing churn and different network sizes. The network size is initiated at 100 member nodes in the first simulation and is later increased to 250 and 500 nodes for the next two simulations, respectively. The number of nodes that undergo churn in our experiments is set to 10,25 and 50 percent of the total number of nodes participating in the network. The number of communication failures are registered and the statistical values for each of the performed experiments are computed. We call this statistic mean number of routing failures and denote it by $R_{n, p}$. The statistic depicts the mean number of routing failures (computed from the 10 attempted communications) per simulated experiment. More formally, we have:

$$
R_{n, p}=\frac{1}{100} \sum_{r=1}^{100} a_{r}
$$

where $a_{r}$ indicates the number of routing failures that were encountered during simulation run $r$ and the corresponding 10 communicating CRDs. The mean number of routing failures $R_{n, p}$ is computed for every simulated network size $n$ together with the corresponding churn percentages $p$.

Figures 2, 3 and 4 show the mean number of routing failures over 100 runs and 10 communications per run for network sizes of 100, 250 and 500 nodes, respectively. Results for both single and multi reality simulations are presented for each of the simulated network sizes. Each experiment is performed with an increasing number of realities, as follows: $1,2,3,4$, 8 . The $95 \%$ confidence intervals for all simulations are within a few percent of the mean.

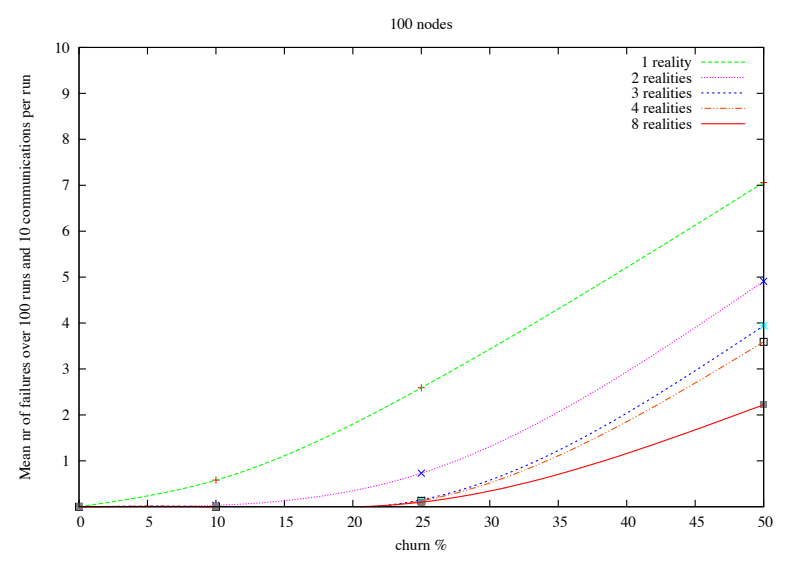

Fig. 2. Mean number of routing failures over 100 runs and 10 communications per run for 100 nodes.

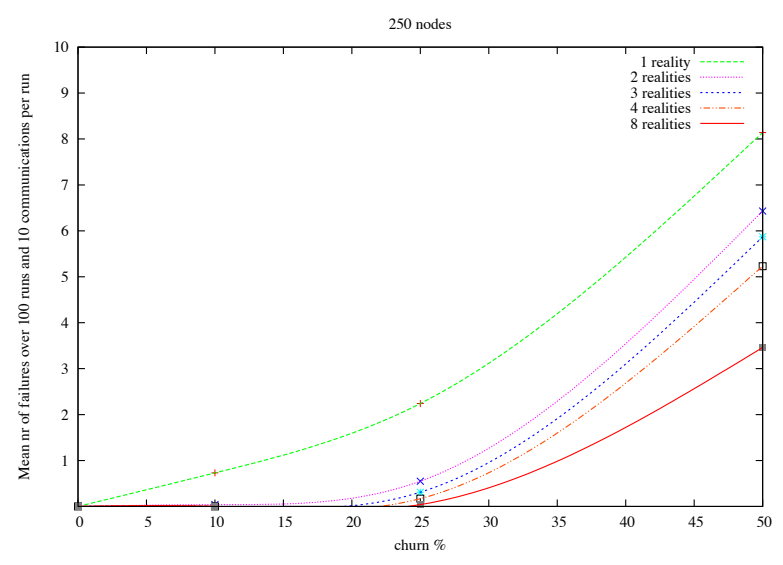

Fig. 3. Mean number of routing failures over 100 runs and 10 communications per run for 250 nodes.

We note that the obtained results are highly dependent on the number of simulated nodes. It is observed that the fault tolerance (resilience to churn) grows with an increasing number of realities. Although, the number of realities needed for an acceptable fault tolerance also depends on the network size, which can observed by comparing the results in figures 24.

Figure 2 shows that for a network size of 100 nodes, a number of 3 realities offers the best tradeoff. In this case, 


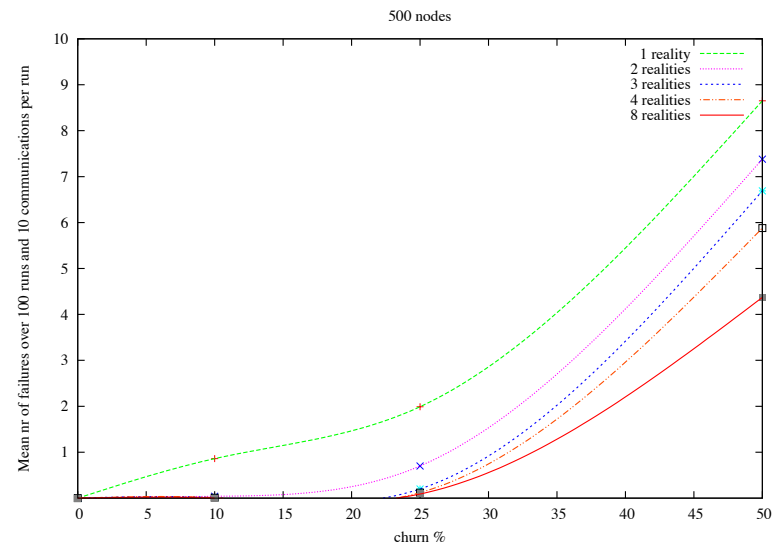

Fig. 4. Mean number of routing failures over 100 runs and 10 communications per run for 500 nodes.

increasing the number of realities to 4 only improves the fault tolerance slightly and consequently does not motivate the increased complexity.

However, in the case of network sizes of 250 (figure 3) or 500 (figure 4) it is observed that a number of at least 4 realities is preferable for an acceptable level of churn resistance. The conclusion therefore is that the number of employed realities has to be increased with a growing number of nodes. Having 50 percent churn in a densely populated network increases the risk of isolating single nodes. In other words, the probability that all the neighbors of a particular node undergo churn at the same time increases for a highly populated network. Naturally, the more populated the network is the more nodes that undergo churn at the same time, for a particular selected churn percentage. Furthermore, this increases the node isolation probability.

In our case, we want to use as few realities as possible though at the same time achieve the best possible fault tolerance. Hence the improved fault tolerance (expressed as percentage) over the single reality is calculated with the formula below and the mean number of failures (over 100 runs and 10 communications per run) from the simulated experiments shown in Table I.

$$
S_{N}=\frac{S_{r, f}}{S_{1, f}}
$$

where $N$ is the number of nodes in the network and $S_{N}$ is the percentage of improved fault tolerance (over the single reality scenario) for experiment with reality number $r$. The number of realities in our simulation spans from 1 to 8 (with the interval $1,2,3,4,8)$, where the percentage of nodes that undergo churn in each experiments is shown by $f$. The simulation results from the 8-reality experiment is used as a reference point for comparing with the rest of the multi-reality simulation scenarios.
Table I shows the mean number of routing failures $R_{n, p}$ for each executed simulation with corresponding realities and node numbers.

\begin{tabular}{|l|l|l|l|l|l|l|}
\hline Nodes & Churn & 1-R & 2-R & $3-\mathrm{R}$ & 4-R & 8-R \\
\hline \multirow{3}{*}{100} & 10 & 0.58 & 0.03 & 0 & 0 & 0 \\
& 25 & 2.59 & 0.73 & 0.13 & 0.1 & 0.10 \\
& 50 & 7.06 & 4.91 & 3.94 & 3.59 & 2.22 \\
\hline \multirow{3}{*}{250} & 10 & 0.73 & 0.04 & 0 & 0 & 0 \\
& 25 & 2.24 & 0.55 & 0.31 & 0.17 & 0.04 \\
& 50 & 8.14 & 6.43 & 5.87 & 5.23 & 3.46 \\
\hline \multirow{3}{*}{500} & 10 & 0.86 & 0.04 & 0.01 & 0.01 & 0 \\
& 25 & 1.99 & 0.70 & 0.20 & 0.12 & 0.09 \\
& 50 & 8.65 & 7.38 & 6.69 & 5.88 & 4.37 \\
\hline
\end{tabular}

TABLE I

MEAN ROUTING FAILURES

The values in table I are collected from each of our simulated multi-reality scenarios, with respect to network size:

As previously mentioned for network sizes of 250 or 500 nodes at least 4 realities are necessary to maintain an acceptable level of churn resilience. However, there is a tradeoff for increasing the number of realities and improving the route computation fault tolerance. This comes at the cost of an increased database size, increased complexity and increased latency for the route computations. Which leads us to conclude that the suggested approach is mainly suitable for static route computations [6], [18], [19].

However, if the licensed bands show very high variations with temporal framings in the order of minutes down to seconds then opportunistic routing needs to be considered. The solutions encompass per-packet dynamic routing over opportunistically available channels, which in other words allows no guarantees to be given for user QoS (Quality of Service) requests. These self-aware routing decisions can on the other hand be made locally by the affected CRDs, allowing instant adaptations to the particular conditions that exist at a specific time [20].

We strive to achieve the best tradeoff between route computation, fault tolerance and increased complexity. The number of realities required for an acceptable fault tolerance to the actual network size must be determined in order to properly dimension the system. Therefore, the scenario displaying values as close as possible to the reference values (of the 8-reality scenario) at a low number of realities is selected, simply for offering the best tradeoff. Having 8 realities or more obviously provides the best fault tolerance though at the cost of high computational latency. In other words, that many realities would not be suitable for real life implementation in a mobile environment, which is the reason we only use it as a reference point.

\section{CONCLUSions}

In this paper we reported on the implementation and performance evaluation of an adapted multi-reality CAN simulator, used as data representation for a CRN architecture. The paper focused on increasing the e2e path computation fault tolerance in the case of massive amounts of churn. The performance 
has been evaluated in terms of mean number of failures per experiment with corresponding realities and node numbers. The tradeoff for increasing the number of realities comes at the cost of an increased database size, increased complexity and increased latency for route computations. Our results allow us to select the best tradeoff between the number of realities and increased complexity for the simulated experiments.

As future work we plan on extending the functionality of our multi-reality approach to use parameters from all CRN dimensions, making it possible to increase the capability of computing paths according to user preferences. At the moment this is done only in terms of shortest distance between source and destination, but can also be achieved from the point of view of all CRN dimensions, allowing for optimizations of, e.g., maximum power output, cost and throughput.

\section{ACKNOWLEDGMENTS}

The authors gratefully acknowledge the support of Euro-NF Network of Excellence, for the work presented in this paper. The work was done as part of the Euro-NF project Networking over Cognitive Radio Networks (NETCO).

\section{REFERENCES}

[1] J. Mitola, III, Cognitive Radio: An Integrated Agent Architecture for Software Defined Radio, PhD thesis, Electrum 204 SE-164 40 Kista, Sweden, 2000.

[2] A. O. Popescu, D. Erman, M. Fiedler, A. P. Popescu, and D. Kouvatsos, "A middleware framework for communication in cognitive radio networks", in ICUMT '10.: International Conference on Ultra Modern Telecommunications, Moscow, Russia, 2010, IEEE Press.

[3] C. Johnson and J. Khalab, "Load based radio resource management for umts picocells", $3 G$ Mobile Communication Technologies, 2003. $3 G$ 2003. 4th International Conference on (Conf. Publ. No. 494), pp. 88 92, jun. 2003.

[4] M. Madfors, K. Wallstedt, S. Magnusson, H. Olofsson, P.-O. Backman, and S. Engstrom, "High capacity with limited spectrum in cellular systems", Communications Magazine, IEEE, vol. 35, no. 8, pp. 38 -45, aug. 1997.

[5] S. Ratnasamy, P. Francis, M. Handley, R. Karp, and S. Shenker, "A scalable content-addressable network", in SIGCOMM '01: Proceedings of the 2001 conference on Applications, technologies, architectures, and protocols for computer communications, New York, NY, USA, 2001, pp. 161-172, ACM.

[6] H. Khalifé, N. Malouch, and S. Fdida, "Multihop cognitive radio networks: to route or not to route", IEEE Network: The Magazine Of Global Internetworking, vol. 23, no. 4, pp. 20-25, 2009.

[7] P.B. Godfrey, S. Shenker, and I. Stoica, "Minimizing churn in distributed systems", in SIGCOMM '06: Proceedings of the 2006 conference on Applications, technologies, architectures, and protocols for computer communications, New York, NY, USA, 2006, pp. 147-158, ACM.

[8] S. Rhea, D. Geels, T. Roscoe, and J. Kubiatowicz, "Handling churn in a dht", in ATEC '04: Proceedings of the annual conference on USENIX Annual Technical Conference, Berkeley, CA, USA, 2004, pp. 10-10, USENIX Association.

[9] A. Binzenhöfer and K. Leibnitz, "Estimating churn in structured p2p networks", in ITC20'07: Proceedings of the 20th international teletraffic conference on Managing traffic performance in converged networks, Berlin, Heidelberg, 2007, pp. 630-641, Springer-Verlag.

[10] A. Popescu, D. Erman, M Fiedler, and D. Kouvatsos, "Routing in content addressable networks: Algorithms and performance", in 20th ITC-Specialist Seminar. 2009, IEEE.

[11] D. Ilie and A. Popescu, "A framework for overlay qos routing", 4th Euro-FGI Workshop on New Trends in Modelling, Quantitative Methods and Measurements, 2007, http://www.bth.se/fou/Forskinfo.nsf/ Sok/6d5f6f18243f6433c125730e006eaa15!OpenDocument.
[12] P. Van Mieghem and F. A. Kuipers, "Concepts of exact qos routing algorithms", IEEE/ACM Transactions on Networking (TON), vol. 12, no. 5, pp. 851-864, 2004.

[13] A. P. Engelbrecht, Fundamentals of Computational Swarm Intelligence, John Wiley \& Sons, 2006.

[14] M. Cai, M. Frank, J. Chen, and P. Szekely, "Maan: A multi-attribute addressable network for grid information services", in Fourth International Workshop on Grid Computing, 2003, pp. 184-191.

[15] M. Subhash, N. Yuan, and S. Chong, "Range and knn searching in p2p", 2004, http://www.comp.nus.edu.sgl ooibc/courses/cs6203/ RangeandkNN.pdf.

[16] P. Ganesan, B. Yang, and H. Garcia-Molina, "One torus to rule them all: multi-dimensional queries in $\mathrm{p} 2 \mathrm{p}$ systems", in 7th International Workshop on the Web and Databases, New York, NY, USA, 2004, pp. 19-24, ACM.

[17] P. Bahl, R. Chandra, T. Moscibroda, R. Murty, and M. Welsh, "White space networking with wi-fi like connectivity", ACM SIGCOMM Computer Communication Review, vol. 39, no. 4, pp. 27-38, 2009.

[18] I.A. Akyildiz, L. Won-Yeol, and K. Chowdhury, "Spectrum management in cognitive radio ad hoc networks", IEEE Network, vol. 23, no. 4, pp. 6-12, 2009.

[19] I.A. Akyildiz, L. Won-Yeol, and K. Chowdhury, "Crahns: Cognitive radio ad hoc networks", Ad Hoc Networks, vol. 7, no. 5, pp. 810-836, 2009.

[20] Erol Gelenbe, "Steps toward self-aware networks", Communications of the ACM, vol. 52, no. 7, pp. 66-75, 2009. 